\section{(A) Check for updates}

Cite this: Org. Biomol. Chem., 2019, 17,8611

Received 26th June 2019

Accepted 9th September 2019

DOI: $10.1039 / c 90 b 01440 c$

rsc.li/obc

\title{
Effects of nano-confinement and conformational mobility on molecular imprinting of cross-linked micelles $\dagger$
}

\begin{abstract}
Kaiqian Chen and Yan Zhao (iD *
Molecular imprinting is a facile method to create guest-complementary binding sites in a cross-linked polymeric network. When performed within cross-linked micelles, the resulting molecularly imprinted nanoparticles (MINPs) exhibited an extraordinary ability to distinguish subtle structural changes in the guest, including the shift of a hydrophilic or hydrophobic group by 1 carbon and addition of a single methylene/methyl group. A high surface-cross-linking density prior to core-cross-linking was key to the high-fidelity imprinting, enhancing both the binding affinity of the imprinted micelle for the template and selectivity among structural analogues. Whereas the imprinted site closely complemented the hydrophilic surface anchoring group and rigid hydrophobic aromatic core, it was expanded significantly for a conformationally mobile small group (i.e., methoxy).
\end{abstract}

\section{Introduction}

Molecular imprinting is a conceptually simple and yet extremely powerful technique to create binding sites for targeted molecules. ${ }^{1,2}$ The traditional method involves polymerization of a mixture of template molecules that are often the interested analytes or their surrogates, functional monomers (FMs) that can interact with the templates through noncovalent or reversible covalent bonds, cross-linkers, and an inert solvent as porogen. Binding sites form spontaneously around the templates as the reaction mixture undergoes polymerization and cross-linking to afford a macroporous polymeric network.

Molecularly imprinted polymers (MIPs) have advanced enormously since their inception and can be generated for different-sized molecules ${ }^{1,2}$ and even large entities such as viruses and bacteria. ${ }^{3-8}$ Ingenious methods have been developed to make the binding sites more accessible and homogenous. ${ }^{9-12}$ Imprinted materials in recent years continue to find new applications in separation, sensing, and catalysis. ${ }^{13-24}$

Our group developed a method to perform molecular imprinting in the micelles of doubly cross-linkable surfactant 1. ${ }^{25}$ The method involves surface-cross-linking of mixed micelles with diazide 2 by the click reaction, ${ }^{26}$ surface functionalization with monoazide 3 by another round of click reaction, ${ }^{27}$ and

Department of Chemistry, Iowa State University, Ames, Iowa 50011-3111, USA. E-mail: zhaoy@iastate.edu; Fax:+1-515-294-0105; Tel: +1-515-294-5845

$\dagger$ Electronic supplementary information (ESI) available: Experimental details, fluorescence titration curves, and additional figures. See DOI: 10.1039/ c9ob01440c core-cross-linking by radical polymerization. ${ }^{25}$ Unlike traditional MIPs, the resulting molecularly imprinted nanoparticles (MINPs) are water-soluble and resemble proteins in their size, hydrophilic exterior, and hydrophobic core. The method allows us to imprint directly in water and also to control the number of binding sites per nanoparticle by the surfactant/template ratio. ${ }^{25}$

MINPs were found to have an exceptional ability to recognize templates among closely related analogues. Isoleucine and leucine, for example, in isomeric di- and tripeptides that differed in the position of a single methyl group by 1 carbon showed $>1 \mathrm{kcal} \mathrm{mol}^{-1}$ difference in binding free energy. ${ }^{28}$ Structurally similar nonsteroidal anti-inflammatory drugs (NSAIDs) displayed cross-reactivities toward their MINP receptors similar to those toward polyclonal antibodies. ${ }^{29}$ Slight variation in the hydrogen-bonding patterns of folic acid derivatives were detected with ease. ${ }^{30}$

Herein, we report a systematic study to understand the origin of the high-fidelity imprinting within cross-linked micelles. The ability to differentiate closely related structural analogues was found to depend critically on the surface-crosslinking of the micelles. In addition, micellar imprinting had different abilities to replicate different parts of the template in the imprinted site. Ionic groups and rigid hydrophobic moieties were much closely imprinted than small groups with high conformational mobility.

\section{Results and discussion}

To gain insight into the micellar imprinting process, we studied the imprinting and binding of a series of closely 
related naphthalene sulfonates (4-11). These compounds are all fluorescent, making it convenient to study their binding by fluorescence titration. They differ subtly in structure, sometimes by the addition or removal of a single methyl or methoxy, other times by the shift of one substituent (hydrophobic or hydrophilic) by 1 carbon. The structural subtlety was meant to test the limit of molecular recognition of MINPs and identify the strongest contributors to the imprinting and binding.

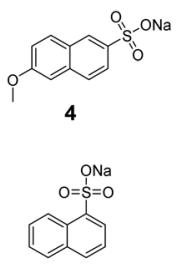

8

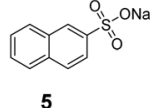

5

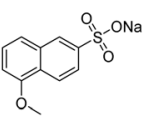

9
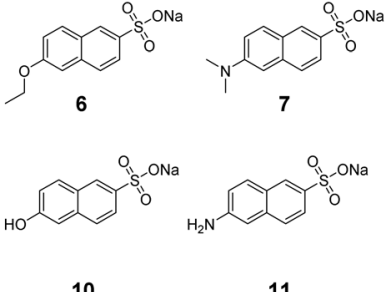

11
In typical MINP preparation, molecular imprinting takes place mostly in the free radical polymerization/cross-linking of the micellar core around the template molecule. Since this step occurs in the confinement of the surface-cross-linked micelle (SCM) formed by the click reaction with diazide 2 , we decided to vary the amount of 2 used in the surface-cross-linking (step 1 in Scheme 1). Under normal condition, 1.2 equivalents of 2 are used relative to the cross-linkable surfactant $1 .^{31}$ The stoichiometry is chosen to afford a reasonably high level of surface-cross-linking while leaving many residual alkyne groups for surface decoration with 3 . The latter step is needed to enhance the hydrophilicity of the MINP, as well as to facilitate its recovery and purification. With the sugar-derived ligand poorly soluble in common organic solvents, we can precipitate MINPs by pouring the aqueous reaction mixture into acetone and remove the templates by washing with acetone/ water and methanol.

For comparison, we varied the amount of the surface-crosslinker from 0 to 1.2 equivalents to that of the cross-linkable surfactant in step 1 in Scheme 1 . These amounts translate to 0 ,

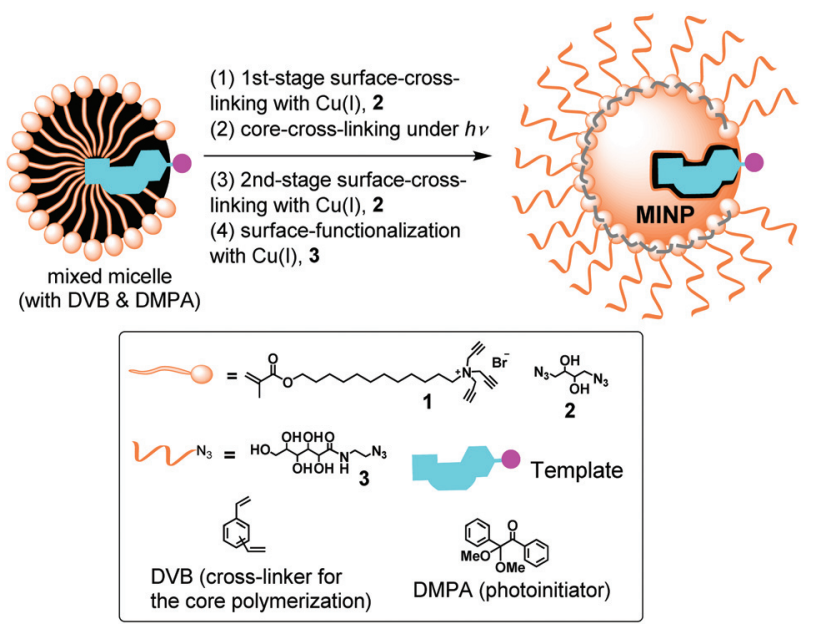

Scheme 1 Preparation of MINP through micellar imprinting.
$33,50,67$, and $100 \%$ of the "normal" amount of 2 in the MINP preparation. After core-cross-linking (step 2), we performed a second-stage surface-cross-linking using 1.2-0 equivalents of 2, respectively, for the MINPs so that the overall amount of surface-cross-linker stayed the same (i.e., 1.2 equivalents) in all cases. In the fourth and final step, the MINPs were functionalized with surface ligand 3. In all the preparations, a 50:1 surfactant/template ratio was used as it is typically done to control the number of binding sites on the micelle. ${ }^{25}$ Because typical MINPs according to dynamic light scattering (DLS) have $\sim 50$ cross-linked surfactants, a 50:1 surfactant/template ratio translates to an average of 1 binding site per nanoparticle. ${ }^{32}$

Table 1 shows that larger particles were obtained at a lower 1st-stage cross-linker level (1CLL), up to $8.84 \mathrm{~nm}$ when no surface-cross-linking was done prior to the free radical corecross-linking. The size decreased generally with increasing $1 \mathrm{CLL}$ and approached the typical size $(\sim 5 \mathrm{~nm})$ when 0.6 equiv. of 2 was used in the first-stage, i.e., $50 \%$ of the normal amount. These results suggest that, to capture the micelle close to the original size, a high surface-cross-linking density is necessary.

More importantly, $\operatorname{MINP}(4)$, i.e., MINP prepared with 4 as the template, displayed stronger binding for the template as 1CLL increased, with the binding constant $\left(K_{\mathrm{a}}\right)$ going up by nearly 6-fold from $2.90 \times 10^{5} \mathrm{M}^{-1}$ to $16.37 \times 10^{5} \mathrm{M}^{-1}$. Thus, even though the total amount of 2 stayed the same in all the MINPs, a higher 1CLL, which led to better formation of SCM, correlated with a stronger imprinting effect in the final MINP.

Binding constants obtained by fluorescence titration has been confirmed in our hands multiple times by isothermal titration calorimetry (ITC), ${ }^{25,28,29}$ which is considered one of the most reliable methods to study intermolecular interactions. ${ }^{33}$ ITC measures the heat change during titration, and affords a wealth of information for the binding, including the binding enthalpy $(\Delta H)$ and number of binding sites per nanoparticle $(N)$, in addition to the binding constant. The binding constants from ITC are shown in parentheses in Table 1 and, as expected, showed good agreements with those obtained from fluorescence titration. Since fluorescence titrations were

Table 1 Sizes and binding constants of MINP(4)s prepared under different cross-linking conditions ${ }^{a}$

\begin{tabular}{llll}
\hline & $\begin{array}{l}{[2] /[1] \text { in the first }+} \\
\text { second-stage } \\
\text { Entry }\end{array}$ & & \\
& surface-cross-linking & $D(\mathrm{~nm})$ & $K_{\mathrm{a}}\left(\times 10^{5} \mathrm{M}^{-1}\right)$ \\
\hline 1 & $0.0+1.2$ & $8.84 \pm 0.40$ & $2.90 \pm 0.18(3.22 \pm 0.39)$ \\
2 & $0.4+0.8$ & $6.61 \pm 0.26$ & $3.33 \pm 0.34(3.80 \pm 0.61)$ \\
3 & $0.6+0.6$ & $5.89 \pm 0.19$ & $7.17 \pm 0.44(6.50 \pm 0.80)$ \\
4 & $0.8+0.4$ & $5.23 \pm 0.21$ & $10.50 \pm 1.96(9.07 \pm 1.29)$ \\
5 & $1.2+0.0$ & $5.57 \pm 0.21$ & $16.37 \pm 3.49(11.80 \pm 2.23)$
\end{tabular}

${ }^{a}$ The binding constants for 4 were determined by fluorescence titrations in triplicates in $10 \mathrm{mM}$ HEPES buffer $(\mathrm{pH} 7.0)$ at $25^{\circ} \mathrm{C}$. The numbers in parentheses were the binding constants determined by ITC under the same conditions. 
much quicker to perform, we used them for the rest of the study.

Guest 5 misses the methoxy group on the C6 of the naphthyl. Because this guest cannot fully occupy the imprinted binding site created from 4, some "high-energy" water molecules will be left in the hydrophobic binding site, near where the methoxy group was during the imprinting. Binding understandably was weaker toward all the $\operatorname{MINP}(4) \mathrm{s}$, regardless of 1CLL (Table 2). The cross-reactivity ratio (CRR), defined as the binding constant of a guest relative to that of template $\mathbf{4}$, decreased from 0.45 to 0.25 , indicating a better binding selectivity for the MINP at higher 1CLLs. The results again support the positive correlation between 1CLL and the molecular imprinting.

Guests 6 and 7 had an extra methylene or methyl group compared to template $\mathbf{4}$, if the O-to-N exchange was not counted. Different from 5, their binding by $\operatorname{MINP}(4)$ started out slightly stronger than the template itself, when no surfacecross-linking was performed prior to the free radical corecross-linking (Table 2, entry 1). Since some imprinting effect was observed for this particular MINP toward guest 5, the stronger binding of 6 and 7 in comparison to 4 should come from a stronger hydrophobic driving force for these guests to enter a flexible, hydrophobic imprinted site. ${ }^{25}$ The results suggest that the imprinted binding site at low 1CLL had considerable plasticity, being able to accommodate an extra methylene/methyl. Since CRR decreased monotonously for $\mathbf{6}$ and 7, a high 1CLL is needed for high binding selectivity for MINP.

Because the size, shape, and distribution of hydrophilic/ hydrophobic groups are fairly similar for 4-7, as better binding sites are formed for template $\mathbf{4}$ at higher 1CLLs, all the analogues displayed stronger binding for $\operatorname{MINP}(4)$. The increase was the largest, however, for the template itself (5.6 vs. 2.1-3.1-fold), consistent with successful molecular imprinting. The trend was shown more clearly in Fig. 1a, which plots the binding constants of 4-7 against the amounts of surfacecross-linker (2) used in the MINP preparation. The data indicates that our MINP was able to detect the missing of a methoxy and the addition of a single methylene/methyl group reasonably well, particularly when prepared with a high level of surface-cross-linker in the first stage. Also, the addition of a

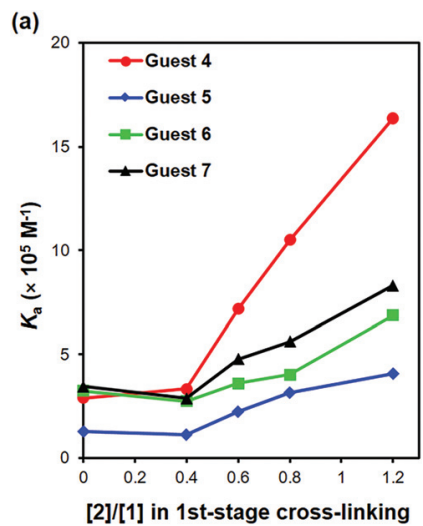

(b)

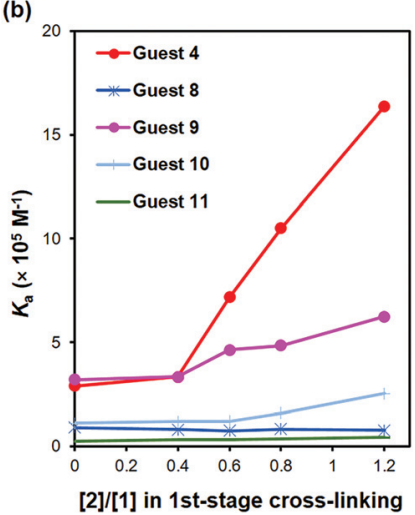

Fig. 1 Binding constants of different guests to MINP(4) prepared with various degrees of 1st-stage cross-linking. (a) Comparison between 4 and 5-7. (b) Comparison between 4 and 8-11.

methylene/methyl (in 6 and 7) was better tolerated than the removal of the methoxy (in 5).

Table 3 lists both the binding constants and CRRs for guests 8-11 for MINP(4)s prepared with different 1CLLs (see Fig. 1b for visual comparisons). Although the data displayed similar trends, several observations are noteworthy. First, moving the hydrophilic sulfonate group by 1 carbon (in 8) was far less tolerated than moving the hydrophobic methoxy group (in 9), evident from the much weaker binding of 8 over 9. Second, replacing the methoxy with a hydrophilic group (in $\mathbf{1 0}$ and 11) was much more detrimental to the binding than

Table 2 Binding data of different guests to MINP(4)s prepared under different cross-linking conditions ${ }^{a}$

\begin{tabular}{|c|c|c|c|c|c|c|c|}
\hline \multirow[b]{2}{*}{ Entry } & \multirow{2}{*}{$\begin{array}{l}{[2] /[1] \text { in the first }+ \text { second-stage }} \\
\text { surface-cross-linking }\end{array}$} & \multicolumn{2}{|l|}{ Guest 5} & \multicolumn{2}{|l|}{ Guest 6} & \multicolumn{2}{|l|}{ Guest 7} \\
\hline & & $K_{\mathrm{a}}\left(\times 10^{5} \mathrm{M}^{-1}\right)$ & $\mathrm{CRR}^{b}$ & $K_{\mathrm{a}}\left(\times 10^{5} \mathrm{M}^{-1}\right)$ & $\mathrm{CRR}^{b}$ & $K_{\mathrm{a}}\left(\times 10^{5} \mathrm{M}^{-1}\right)$ & $\mathrm{CRR}^{b}$ \\
\hline 1 & $0.0+1.2$ & $1.30 \pm 0.03$ & 0.45 & $3.24 \pm 0.60$ & 1.12 & $3.46 \pm 0.21$ & 1.19 \\
\hline 2 & $0.4+0.8$ & $1.13 \pm 0.06$ & 0.34 & $2.74 \pm 0.61$ & 0.82 & $2.87 \pm 1.00$ & 0.86 \\
\hline 3 & $0.6+0.6$ & $2.22 \pm 0.07$ & 0.31 & $3.61 \pm 0.05$ & 0.50 & $4.77 \pm 0.77$ & 0.67 \\
\hline 4 & $0.8+0.4$ & $3.17 \pm 0.53$ & 0.30 & $4.03 \pm 1.32$ & 0.38 & $5.62 \pm 1.05$ & 0.54 \\
\hline 5 & $1.2+0.0$ & $4.09 \pm 0.24$ & 0.25 & $6.87 \pm 1.52$ & 0.42 & $8.30 \pm 1.58$ & 0.51 \\
\hline
\end{tabular}

${ }^{a}$ Fluorescence titrations were performed in triplicates in $25 \mathrm{mM}$ HEPES buffer $(\mathrm{pH} 7.0)$ at $25{ }^{\circ} \mathrm{C} .{ }^{b} \mathrm{CRR}$ is the cross-reactivity ratio, defined as the binding constant of a guest relative to that of template 4 . 
Table 3 Binding data of different guests to MINP(4)s prepared under different cross-linking conditions ${ }^{a}$

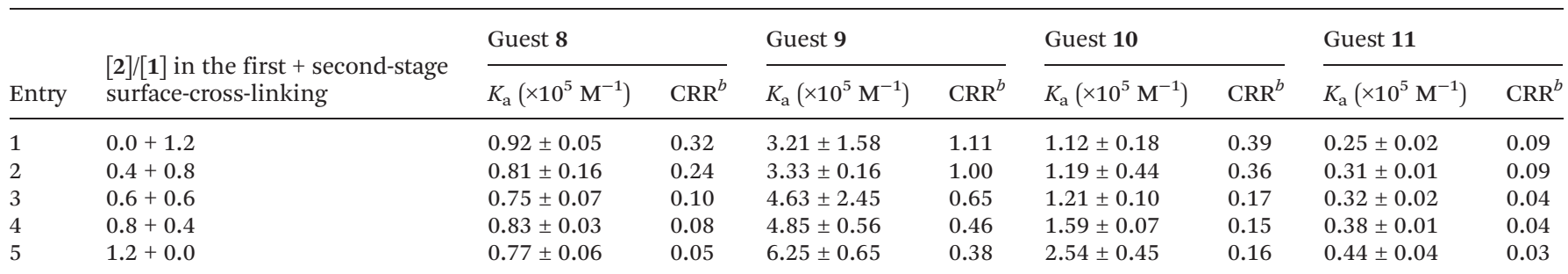

${ }^{a}$ Fluorescence titrations were performed in triplicates in $10 \mathrm{mM}$ HEPES buffer $(\mathrm{pH} 7.0)$ at $25{ }^{\circ} \mathrm{C} .{ }^{b} \mathrm{CRR}$ is the cross-reactivity ratio, defined as the binding constant of a guest relative to that of template 4 .

missing the methoxy (in 5), shifting the position of the methoxy by 1 carbon (in 9), or adding an extra methylene/ methyl group to the methoxy (in 6 and 7 ).

We also used 5 as the template to prepare MINPs, without the methoxy on the naphthalene ring. We then studied the binding of 5, 4, and 9 by MINP(5)s prepared also at different 1CLLs. The binding data are shown in Table 4 and plotted in Fig. 2. The template itself (5) showed the same trend as observed earlier, with an increasing 1CLL leading to stronger binding. The trend supports again the positive correlation between higher 1CLLs and stronger molecular imprinting.

Our earlier data with $\operatorname{MINP}(4)$ showed that imprinted sites formed at lower 1CLLs had significant plasticity. The same observation was made with $\mathbf{4}$ and $\mathbf{9}$. With an extra methoxy group, they had a stronger hydrophobic driving force than template $\mathbf{5}$ to enter a hydrophobic site. As long as the binding site was flexible enough to accommodate these guests, they showed a stronger binding toward MINP(5) (see Table 4 and Fig. 2).

Interestingly, in contrast to everything observed for $\operatorname{MINP}(4)$ in Fig. 1, the binding of $\mathbf{4}$ and $\mathbf{9}$ exhibited a downward profile with increasing 1CLLs (Fig. 2). What could be the reason for the different trends? Our postulation is that the different groups of the templates had different templating effects during micellar imprinting. Template $\mathbf{4}$ has three parts in the structure: a rigid aromatic naphthyl, a conformationally mobile methoxy, and an ionic sulfonate group. Template $\mathbf{5}$ had the methoxy missing. Many of our previous studies showed that large, rigid hydrophobic groups are imprinted well..$^{25,28,29,34,35}$ Fig. $1 \mathrm{~b}$ shows that the hydrophilic anchor (sulfonate group) was also strongly

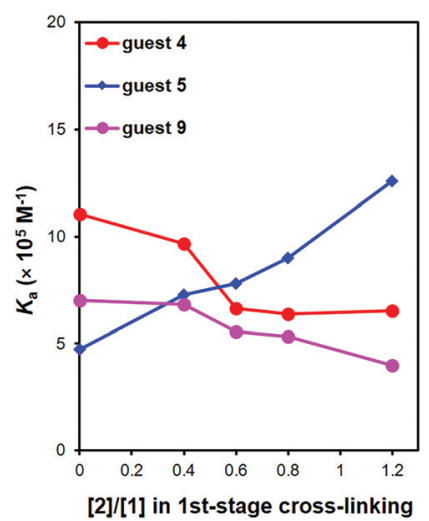

Fig. 2 Binding constants of different guests to MINP(5) prepared with various degrees of 1 st-stage cross-linking.

imprinted. Since the ionic group has to stay on the surface of the micelle to be solvated by water, its relative orientation should be very important to the imprinting and binding, as it determines the topology of the amphiphile.

The methoxy group of $\mathbf{4}$, on the other hand, is quite different. It is small and expected to stay in the hydrophobic region of the micelle, both due to its hydrophobicity and its opposite position to the hydrophilic sulfonate group on the naphthalene ring. Its conformational mobility suggests a rapid rotation around the two $\mathrm{C}-\mathrm{O}$ bonds while the micellar core undergoes polymerization/cross-linking. With these rotations being much faster than the chemical reaction, a larger

Table 4 Binding data of different guests to MINP(5)s prepared under different cross-linking conditions ${ }^{a}$

\begin{tabular}{|c|c|c|c|c|c|c|c|}
\hline 2 & $0.4+0.8$ & $7.28 \pm 1.29$ & 1 & $9.66 \pm 0.01$ & 1.33 & $6.82 \pm 0.00$ & 0.94 \\
\hline 3 & $0.6+0.6$ & $7.80 \pm 3.05$ & 1 & $6.63 \pm 0.01$ & 0.85 & $5.54 \pm 0.48$ & 0.71 \\
\hline 4 & $0.8+0.4$ & $9.01 \pm 3.39$ & 1 & $6.36 \pm 0.00$ & 0.71 & $5.32 \pm 0.08$ & 0.59 \\
\hline 5 & $1.2+0.0$ & $12.57 \pm 0.02$ & 1 & $6.54 \pm 0.33$ & 0.52 & $3.97 \pm 0.21$ & 0.32 \\
\hline
\end{tabular}

${ }^{a}$ Fluorescence titrations were performed in triplicates in $10 \mathrm{mM}$ HEPES buffer $(\mathrm{pH} 7.0)$ at $25{ }^{\circ} \mathrm{C} .{ }^{b} \mathrm{CRR}$ is the cross-reactivity ratio, defined as the binding constant of a guest relative to that of template 5 . 
imprinted space should be created than what its static size would imply.

Once the above point is made clear, it seems very reasonable that $\operatorname{MINP}(4)$ tolerated the size-increase of the methoxy and shift in position reasonably well. As long as the structural analogues (6, 7, and 9) could occupy the same pocket created after $\mathbf{4}$, a better formed imprinted site for the template would lead to stronger binding for the analogues as well (Fig. 1). Without this methoxy group in the template, no "added fuzzy space" could be created in the binding site of $\operatorname{MINP}(5)$. At low 1CLLs, the flexible imprinted site for $\mathbf{5}$ was able to bind the structural analogues (4 and 9) more strongly due to their higher hydrophobicity. At higher 1CLLs, however, the better formed imprinted site lost its ability to reconfigure itself for mismatched guests (4 and 9). Even with a stronger hydrophobic driving force, these guests could not fit into the site very well, thus displaying a downward trend with increasing 1CLL (Fig. 2).

Micellar imprinting has become a powerful method to create synthetic receptors ${ }^{28,36-38}$ and catalysts that operate in water. $^{39,40}$ The most interesting findings of this work is the strong positive correlation between higher 1CLLs and stronger imprinting, as well as the different templating effects of different parts of the template molecule. A higher 1CLL leads to more rigid and smaller SCM, with the latter confirmed by DLS. It could be generally useful that the best imprinting was obtained under such conditions. Several possible reasons might have helped the micellar imprinting. Free radical polymerization and cross-linking in a confined nanospace (of a micelle) could be faster than similar reactions in the bulk. Faster chemical reactions set the polymeric network faster, beneficial to molecular imprinting. The dynamics of the template could be different as well. When the template molecule diffuses out of an SCM, a flexible structure at low surfacecross-linking density could easily reconfigure itself to lower its free energy, via collapsing the hydrophobic tails of the surfactants for example. A more rigid structure would have difficulty doing the same, and thus might tend to keep the template molecule longer within the SCM and enhance the imprinting as a result.

\section{Conclusions}

Systematic evaluation of structurally similar templates/guests confirmed that confining the free radical polymerization/crosslinking in the nanospace of the surface-cross-linked micelle was critical to the binding affinity and selectivity of the MINP receptors. This could be useful to other types of molecular imprinting as well. In addition, our study suggests that the size, shape, and position of imprinted sites correlate strongly with rigid hydrophobic groups and also the hydrophilic anchor. Some level of "fuzziness", however, should be expected for small, conformationally mobile groups such as methoxy. Such flexibility could be used judiciously in the design of molecularly imprinted catalysts, where fast binding of sub- strates and release of products are key parameters to be considered.

\section{Experimental}

\section{General method}

Compounds 5, 8, 10, and $\mathbf{1 1}$ are commercially available and were used as received (purity $>98 \%$ ). All other reagents and solvents were of ACS-certified grade or higher, and were used as received from commercial suppliers. Routine ${ }^{1} \mathrm{H}$ and ${ }^{13} \mathrm{C}$ NMR spectra were recorded on a Bruker DRX-400 or on a Varian VXR-400 spectrometer. ESI-MS mass spectra was recorded on a Shimadzu LCMS-2010 mass spectrometer. Fluorescence spectra were recorded at ambient temperature on a Varian Cary Eclipse Fluorescence spectrophotometer. DLS data were collected on a Malvern Zetasizer Nano ZS.

\section{Synthetic procedures}

Syntheses of compounds $1-3,{ }^{25} 4,{ }^{41} \mathbf{6},{ }^{42} 7^{43}$ and MINP have been reported.

Compound 9. The synthesis followed similar procedures used for 4. $^{41}$ 5-Hydroxynaphthalene-2-sulfonic acid (0.4485 g, $2.0 \mathrm{mmol})$ and sodium hydroxide $(0.16 \mathrm{~g}, 4.0 \mathrm{mmol})$ were dissolved in $9 \mathrm{~mL}$ of water, followed by the addition of dimethyl sulfate $(210 \mu \mathrm{L}, 2.2 \mathrm{mmol})$. After the reaction mixture was stirred at $55{ }^{\circ} \mathrm{C}$ for $1 \mathrm{~h}$, sodium chloride (0.8182 g) was added. The precipitate formed was collected by suction filtration and dried in vacuo to afford an off-white powder (0.2748 g, 53\%). ${ }^{1} \mathrm{H}$ NMR (400 MHz, Methanol- $d^{4}$ ) $\delta$ 8.29-8.24 (m, 2H), 7.84 (dd, $J=8.8,1.8 \mathrm{~Hz}, 1 \mathrm{H}), 7.51-7.43(\mathrm{~m}, 2 \mathrm{H}), 6.98(\mathrm{dd}, J=7.1,1.5 \mathrm{~Hz}$, $1 \mathrm{H}), 4.00(\mathrm{~s}, 3 \mathrm{H}) .{ }^{13} \mathrm{C} \mathrm{NMR}\left(101 \mathrm{MHz}\right.$, methanol- $\left.d^{4}\right) \delta 155.25$, 133.52, 126.80, 126.54, 125.82, 124.67, 121.98, 121.80, 120.42, 105.03, 54.73. ESI-HRMS calcd for $\mathrm{C}_{11} \mathrm{H}_{9} \mathrm{O}_{4} \mathrm{~S}(\mathrm{~m} / \mathrm{z}):[\mathrm{M}-\mathrm{Na}]^{-}$, 237.0227; found, 237.0238 .

\section{Fluorescence titration}

Stock solutions of MINP $(200 \mu \mathrm{M})$ and the guests $(200 \mu \mathrm{M})$ were prepared in $10 \mathrm{mM}$ HEPES buffer ( $\mathrm{pH}$ 7.0). For the titrations, a typical procedure is as follows. An aliquot $(10 \mu \mathrm{L})$ of the guest stock solution was added to $1.99 \mathrm{~mL}$ of the HEPES buffer in a quartz cuvette at room temperature. The concentration of the guest was $1.0 \mu \mathrm{M}$ in every case. The sample was gently vortexed for $30 \mathrm{~s}$ before its fluorescence spectrum was recorded. Aliquots of the MINP solution was added and the spectrum was recorded after each addition. The titration was continued until saturation was reached and the total volume of the MINP solution added was kept below $100 \mu \mathrm{L}$. The binding constant was obtained by nonlinear least squares curving fitting of the emission intensity to the $1: 1$ binding isotherm as described in the ESI. $\dagger$

\section{ITC titration}

ITC was performed using a MicroCal VP-ITC Microcalorimeter with Origin 7 software and VPViewer2000 (GE Healthcare, Northampton, MA). The determination of binding constants 
by ITC followed standard procedures. ${ }^{44-46}$ In general, a solution of an appropriate guest in Millipore water was injected in equal steps into $1.43 \mathrm{~mL}$ of the corresponding MINP in the same solution. The top panel shows the raw calorimetric data. The area under each peak represents the amount of heat generated at each ejection and is plotted against the molar ratio of the MINP to the guest. The smooth solid line is the best fit of the experimental data to the sequential binding of $\mathrm{N}$ binding site on the MINP. The heat of dilution for the guest, obtained by titration carried out beyond the saturation point, was subtracted from the heat released during the binding. Binding parameters were auto-generated after curve fitting using Microcal Origin 7.

\section{Conflicts of interest}

There are no conflicts to declare.

\section{Acknowledgements}

We thank NIGMS (R01GM113883) for financial support.

\section{Notes and references}

1 G. Wulff, Chem. Rev., 2001, 102, 1-28.

2 K. Haupt and K. Mosbach, Chem. Rev., 2000, 100, 24952504.

3 A. Aherne, C. Alexander, M. J. Payne, N. Perez and E. N. Vulfson, J. Am. Chem. Soc., 1996, 118, 8771-8772.

4 K. Ren and R. N. Zare, ACS Nano, 2012, 6, 4314-4318.

5 J. Borovička, S. D. Stoyanov and V. N. Paunov, Nanoscale, 2013, 5, 8560-8568.

6 X. Shen, J. S. Bonde, T. Kamra, L. Bülow, J. C. Leo, D. Linke and L. Ye, Angew. Chem., Int. Ed., 2014, 53, 10687-10690.

7 H. Bao, B. Yang, X. Zhang, L. Lei and Z. Li, Chem. Commun., 2017, 53, 2319-2322.

8 J. Pan, W. Chen, Y. Ma and G. Pan, Chem. Soc. Rev., 2018, 47, 5574-5587.

9 S. C. Zimmerman, M. S. Wendland, N. A. Rakow, I. Zharov and K. S. Suslick, Nature, 2002, 418, 399-403.

10 K. G. Yang, M. M. Berg, C. S. Zhao and L. Ye, Macromolecules, 2009, 42, 8739-8746.

11 X. Shen and L. Ye, Macromolecules, 2011, 44, 5631-5637.

12 F. Canfarotta, A. Poma, A. Guerreiro and S. Piletsky, Nat. Protoc., 2016, 11, 443.

13 D. Lakshmi, A. Bossi, M. J. Whitcombe, I. Chianella, S. A. Fowler, S. Subrahmanyam, E. V. Piletska and S. A. Piletsky, Anal. Chem., 2009, 81, 3576-3584.

14 Y. Hoshino, H. Koide, T. Urakami, H. Kanazawa, T. Kodama, N. Oku and K. J. Shea, J. Am. Chem. Soc., 2010, 132, 6644-6645.

15 Y. Hoshino, T. Kodama, Y. Okahata and K. J. Shea, J. Am. Chem. Soc., 2008, 130, 15242-15243.
16 J. L. Urraca, C. S. A. Aureliano, E. Schillinger, H. Esselmann, J. Wiltfang and B. Sellergren, J. Am. Chem. Soc., 2011, 133, 9220-9223.

17 Y. Ma, G. Q. Pan, Y. Zhang, X. Z. Guo and H. Q. Zhang, Angew. Chem., Int. Ed., 2013, 52, 1511-1514.

18 T. Kuwata, A. Uchida, E. Takano, Y. Kitayama and T. Takeuchi, Anal. Chem., 2015, 87, 11784-11791.

19 Y. B. Liu, S. M. Fang, J. Q. Zhai and M. P. Zhao, Nanoscale, 2015, 7, 7162-7167.

20 J. Liu, D. Yin, S. Wang, H. Y. Chen and Z. Liu, Angew. Chem., Int. Ed., 2016, 55, 13215-13218.

21 R. Horikawa, H. Sunayama, Y. Kitayama, E. Takano and T. Takeuchi, Angew. Chem., Int. Ed., 2016, 55, 13023-13027.

22 M. Panagiotopoulou, Y. Salinas, S. Beyazit, S. Kunath, L. Duma, E. Prost, A. G. Mayes, M. Resmini, B. T. S. Bui and K. Haupt, Angew. Chem., Int. Ed., 2016, 55, 8244-8248.

23 M. Bertolla, L. Cenci, A. Anesi, E. Ambrosi, F. Tagliaro, L. Vanzetti, G. Guella and A. M. Bossi, ACS Appl. Mater. Interfaces, 2017, 9, 6908-6915.

24 G. Pan, S. Shinde, S. Y. Yeung, M. Jakštaitè, Q. Li, A. G. Wingren and B. Sellergren, Angew. Chem., Int. Ed., 2017, 56, 15959-15963.

25 J. K. Awino and Y. Zhao, J. Am. Chem. Soc., 2013, 135, 12552-12555.

26 Y. Zhao, Langmuir, 2016, 32, 5703-5713.

27 X. Li and Y. Zhao, Langmuir, 2012, 28, 4152-4159.

28 J. K. Awino, R. W. Gunasekara and Y. Zhao, J. Am. Chem. Soc., 2017, 139, 2188-2191.

29 J. K. Awino and Y. Zhao, ACS Biomater. Sci. Eng., 2015, 1, 425-430.

30 L. Duan and Y. Zhao, Bioconjugate Chem., 2018, 29, 1438-1445. 31 Note that the highest surface-cross-linking density is reached with 1.5 equivalents of 2 , because of the $3: 2$ ratio in the number of reactive groups on $\mathbf{1}$ and 2.

32 In this particular work, because the size (and molecular weight) of MINP changed with the 1st-stage cross-linker level (Table 1), the true number of binding sites per nanoparticle differed for different MINPs. For comparison purposes, a nominal molecular weight of 50000 was used for all the MINPs, based on $\sim 50$ units of cross-linked monomer, with each unit (MW 959.7) defined as $1 \times 1$ (MW 464.5), $1.2 \times 2$ (MW 172.1), $0.6 \times 3$ (MW 264.1), and 1 $\times$ DVB (MW 130.2). Since a 50:1 ratio of surfactant/template was used in all the MINP preparations, the $1: 1$ binding model for the curve fitting was for the binding between the guest and a nominal MINP consisting of 50 units of the cross-linked monomer.

33 F. P. Schmidtchen, in Supramolecular Chemistry: From Molecules to Nanomaterials, ed. J. W. Steed and P. A. Gale, Wiley, Online, 2012, DOI: 10.1002/9780470661345.smc024.

34 J. K. Awino and Y. Zhao, Chem. Commun., 2014, 50, 57525755.

35 M. D. Arifuzzaman and Y. Zhao, J. Org. Chem., 2016, 81, 7518-7526.

36 J. K. Awino, R. W. Gunasekara and Y. Zhao, J. Am. Chem. Soc., 2016, 138, 9759-9762. 
37 R. W. Gunasekara and Y. Zhao, J. Am. Chem. Soc., 2017, 139, 829-835.

38 Y. Zhao, Chem. - Eur. J., 2018, 24, 14001-14009.

39 M. Arifuzzaman and Y. Zhao, ACS Catal., 2018, 8, 81548161.

40 S. Fa and Y. Zhao, Bioorg. Med. Chem. Lett., 2019, 29, 978981.

41 M. F. Schmidt, O. Korb, N. I. Howard, M. V. B. Dias, T. L. Blundell and C. Abell, ChemMedChem, 2013, 8, 54-58.
42 R. Fleischhauer, Tris- and Polyazo Dyes, DE943662, 1956.

43 S. Pan, S.-Y. Jang, S. S. Liew, J. Fu, D. Wang, J.-S. Lee and S. Q. Yao, Angew. Chem., Int. Ed., 2018, 57, 579-583.

44 T. Wiseman, S. Williston, J. F. Brandts and L. N. Lin, Anal. Biochem., 1989, 179, 131-137.

45 I. Jelesarov and H. R. Bosshard, J. Mol. Recognit., 1999, 12, 3-18.

46 A. Velazquez-Campoy, S. A. Leavitt and E. Freire, Methods Mol. Biol., 2004, 261, 35-54. 